\title{
The Most Effective Number of Elastic Taping Applications on the Muscle Activity and Maximum Peak of the Wrist Extensor Muscle in Patients with Stroke
}

\author{
Ju Chul Cho, Byoung Kwon Lee, Seung Chul Chon \\ Department of Physical Therapy, College of Medical Science Konyang University, Daejeon, 302-832
}

\section{Corresponding Author \\ Seung Chul Chon \\ Department of Physical Therapy, College of Medical Science Konyang University, \\ Daejeon, 302-832 \\ Mobile : +82-10-8615-4767 \\ Email : biomechanic1@hotmail.com}

Received : October 15, 2014

Revised : October 25, 2014

Accepted: November 14, 2014
Objective: The aim of this study is to evaluate the differences in electromyographic activities of upper extremity muscle between repeated taping and to compare the effects of each taping method in stroke patients.

Background: Taping studies for functional improvement and pain relief of the UE have been conducted using various methods. Despite being an important factor when you treatment to a patient in a clinical attachment numbers situation quantitative research is that there is not at all to the taping at the time of application.

Method: Twenty patients volunteered in this study and were tested under four taping conditions as follows, in random order: (1) no taping, (2) taping applied once, (3) taping applied twice, and (4) taping applied thrice. The muscle activity and maximum peak of the extensor carpi radialis muscle in electromyographic activities were measured, respectively.

Results: The muscle activity and maximum peak of the extensor carpi radialis muscle showed significant differences among the four conditions $(p<.05)$. In the post hoc test, the extensor muscle showed significant differences in muscle activity and maximum peak in electromyographic activities, except between taping applied twice and thrice.

Conclusion: These findings demonstrate that repeated taping up to two times may be useful in improving the muscle activity and maximum peak of the extensor muscle.

Application: This study provides useful information to future researchers regarding the effects of repeated taping applications on muscle activity and maximum peak of the muscles of the human body.

Keywords: Muscle activity, Number of application, Stroke, Taping, Upper extremity

\section{Introduction}

The upper extremity (UE) function of stroke patients plays a highly important role in their activities of daily living (ADL), is considered to be recoverable through rehabilitation, and is kinematically composed of parts of the body such as the elbow and wrist joints, and finger(Bartolo et al., 2014; Smith et al., 2009). However, if 
neuromuscular functional deficits of the UE cause a problem to its movement pattern (Buckle and Devereux, 2002), they can sequentially lead to dysfunction and pain, which can affect patients' ADL (Haudum et al., 2012). Among the proper functions of the activity of the $U E$, extension of the wrist is necessary for the execution of various $A D L$ and the complex function of the hand (Van Elk et al., 2004). In particular, weakness and abnormal tone of muscle are major problems in ADL. Consequently, various methods have been developed to enable stroke patients to return to independent living and to aid in their functional recovery (Jaraczewska and Long, 2006).

Among these methods is the use of the elastic tape (ET), which is primarily designed to allow easy adhesion and strong elasticity, and is maintained as a reconditioner of the musculoskeletal system. As a non-drug therapy, it has shown good results in the preventive management of injury, pain relief, and muscle strengthening in various musculoskeletal disorders (Firth et al., 2010). In addition, the ET has been widely used for edema, muscle imbalance, and motor control associated with functional activity of patients with neurological problem such as those with stroke, as well as in rehabilitation methods (Bell and Muller, 2013; Montalvo et al., 2014).

ET has been widely used to adjust muscle contraction and relaxation, enhance movement, and decrease pain (Montalvo et al., 2014). It is believed to lead to muscle recovery in various areas of the body. A receptor called Golgi tendon organ and muscle spindle exists in the muscle. Adjusting in response to the tension of muscle contraction in the mutual cooperation of these effects does not seem to cause any muscle damage, but it becomes a functional problem when muscle balance is altered, internal pressure is generated in the soft tissue (muscles, ligaments, and tendons), and the blood and lymphatic circulations are disturbed (Alexander et al., 2008; Karadag-Saygi et al., 2010). Therefore, in theory, to reduce the inflammation and tension of the muscles and joints, the rearranged muscle film should be returned to normal muscle function during muscle contraction and thus prevent pain (Mostafavifar et al., 2012).

Although taping is believed to be useful in basic rehabilitation of hemiplegic patients, case studies of taping therapy applied to stroke patients are rare. The application of tape to the joints of the paralyzed side of stroke patients has been reported to be effective in improving motor function and reducing pain (Jaraczewska and Long, 2006). The application of tape was also found to have a positive effect on shoulder symmetry and shoulder joint pain in adult hemiplegic patients (Pandian et al., 2013). However, other studies did not report visible improvements in muscle strength or ADL after taping therapy (Parreira et al., 2014). We applied tape to the muscles of healthy subjects and evaluated the impact on their wrist extension and muscle strength. We found little difference after the application in all the joints in terms of changes in wrist muscle strength and grip strength. Therefore, intensive study of the benefits of taping is needed.

Taping studies for functional improvement and pain relief of the UE have been conducted using various methods. Despite being an important factor when you treatment to a patient in a clinical attachment numbers situation quantitative research is that there is not at all to the taping at the time of application. Therefore, in this study, we examined the effect of repeated taping applications to the forearm of stroke patients on wrist extension using surface electromyography (EMG). The hypothesis of the present study was that the muscle activity during wrist extension would be significantly reduced when taping is applied once at the attachment site, as compared to applying it two or three times.

\section{Method}

\subsection{Subjects}

Twenty stroke patients (age: $63.06 \pm 10.99$ years, height: $162.89 \pm 8.25 \mathrm{~cm}$, and weight: $63.74 \pm 9.49 \mathrm{~kg}$ ) were recruited from a university hospital. The inclusion criteria for the subjects were as follows: 
(1) Stroke diagnosed by a specialist through magnetic resonance imaging

(2) $\geq 24$ points on the mini-mental state examination, a cognitive function test tool

(3) Ability to understand simple verbal instructions and behavior

(4) No limitation of motion of the wrist joint

(5) A modified Ashworth scale grade of $0 \sim 1$

(6) A manual muscle test grade of fair or better

(7) Absence of sensory problems. Patients with congenital malformations of the UE, neurological disorders, and musculoskeletal system disorders were excluded. All the subjects were given a sufficient explanation of the research

\subsection{Surface electromyographic recording}

The surface EMG myosystem (Noraxon, Noraxon Inc., AZ, USA), which comprised 8-channels, an analog to digital converter with 16-bit resolution, and a universal serial bus connection, was used to assess muscle activity and maximum peak for the extensor carpi radialis (ECR) muscle. The skin was cleaned with alcohol, and a razor was used to remove hair. Electrodes with electrolyte gel were used to reduce the skin resistance during the surface EMG measurements. After the skin was dry, pairs of circular $\mathrm{Ag} / \mathrm{AgCl}$ surface electrodes (Noraxon, Noraxon Inc., AZ, USA) with a contact diameter of $11 \mathrm{~mm}$ were placed on standard locations, as suggested by Cram et al. (1998), with an inter-electrode distance of $15 \mathrm{~mm}$. The electrode was attached to the belly muscle $5 \mathrm{~cm}$ from the lateral epicondyle of the elbow, parallel to the muscle fibers where the electrodes of the ECR were mounted. We determined the dominant UE for each participant by observing the limb with which each participant grabbed a pen. The sampling rate of the EMG signal was $1,024 \mathrm{~Hz}$, and a $60-\mathrm{Hz}$ notch filter was used for noise removal. Bandpass filtering was applied at 25$500 \mathrm{~Hz}$. The subjects were instructed to remove their necklaces, watches, or rings prior to the EMG measurements.

To normalize the EMG signals recorded for ECR muscle, the root mean square (RMS) of a 5-sec maximal voluntary isometric contraction (MVIC) was calculated at the manual muscle testing positions recommended by Kendall et al. (2005) and expressed as a percentage of the calculated RMS of the \%MVIC. EMG data for each muscle were averaged for the three trials with a 3min rest interval.

\subsection{Procedure}

The subjects were tested under the following four taping conditions of the ECR muscle, in random order: (1) no taping, (2) taping applied once, (3) taping applied twice, and (4) taping applied thrice. To determine the order of application of each condition, one person who was not involved in the assessment of the subjects performed the conventional randomization directory process in which a random number board was used to assign one card to each subject. The guidelines for taping each muscle were consistent with the protocol suggested by Jaraczewska and Long (2006). A standard tape (kinesiology taping, 3NS, Seoul, Korea) with a diameter of $5 \mathrm{~cm}$ was applied on the dominant UE for all applications across the four taping conditions. The tape length for all the taping conditions was adjusted for the circumference of the application regions, and the interval between the origin and insertion points of each patient, respectively. The middle portion of the tape was dedicated for the placement of the electrodes.

EMG data were collected with the subjects sitting on a fixed chair with a backrest, with the hip and knee joints flexed to $90^{\circ}$, elbow flexed to $90^{\circ}$, and wrist in neutral position, looking straight ahead (Figure 1). The EMG measurement for measuring the activity of the ECR muscle, the UE was positioned with the shoulder flexed to $15^{\circ}$, the elbow flexed to $90^{\circ}$, the forearm supinated, and the wrist extended to $10^{\circ}$. An isometric contraction of the UE was then performed for $5 \mathrm{sec}$. EMG data were averaged for the three trials with a 1-min rest interval. All the experiments were performed in the same environment. The measurements were performed before and after the experiment. The correct orientation was maintained during the testing of the subjects on the 
side with the damage.



Figure 1. The experimental equipment and position of EMG measurement

\subsection{Statistical analysis}

The SPSS 18.0 program (SPSS Inc., Chicago, IL, USA) was used to analyze the differences in the muscle activity of the ECR according to the number of times the tape was applied. The differences were compared using the repeated one-way analysis of variance and post hoc analysis with Bonferroni correction. Statistical significance was set at $p=.05$.

\section{Results}

This section summarizes the data on the muscle activity and maximum peak of the ECR muscle during the 4 taping conditions. The muscle activities and maximum peak appeared to be significantly different between the 4 taping conditions (no taping, taping applied once, taping applied twice, and taping applied thrice) $(p=.000)(p=.000)$ (Table 1).

Table 1. The muscle activity and maximum peak of ECR muscle during the 4 taping conditions

\begin{tabular}{l|l|l|l|c|c}
\hline & No taping & One taping & Two taping & Three taping & $F$ \\
\hline Muscle activity & $60.70 \pm 7.89^{a}$ & $51.53 \pm 7.41$ & $44.49 \pm 6.53$ & $44.27 \pm 6.22$ & $15.28^{\star}$ \\
\hline Maximum peak & $118.7 \pm 13.62$ & $105.1 \pm 12.11$ & $93.8 \pm 12.62$ & $90.6 \pm 12.33$ & $12.07^{\star}$ \\
\hline
\end{tabular}

amean \pm standard deviation, ${ }^{*} p<.05$

In the post hoc test, the activities of the ECR muscle showed significant differences between the no taping and applying taping once conditions $(p=.000)$, between the no taping and applying taping twice conditions $(p=.000)$, between the no taping and applying taping thrice conditions $(p=.000)$, between the applying taping once and applying taping twice conditions $(p=.001)$ and between applying taping once and applying taping thrice conditions $(p=.002)$, but not between the applying taping twice and thrice conditions $(p=1.000)$ (Figure 2).

In the post hoc test, the maximum peak of the ECR muscle showed significant differences between no taping and applying 
taping twice conditions $(p=.000)$, between no taping and the three taping conditions $(p=.000)$, between applying taping once and twice conditions $(p=.018)$, and between applying taping once and thrice conditions $(p=.026)$, but not between the no taping and applying taping once conditions $(p=.121)$ or between the applying taping twice and thrice conditions $(p=1.000)$ (Figure 2).
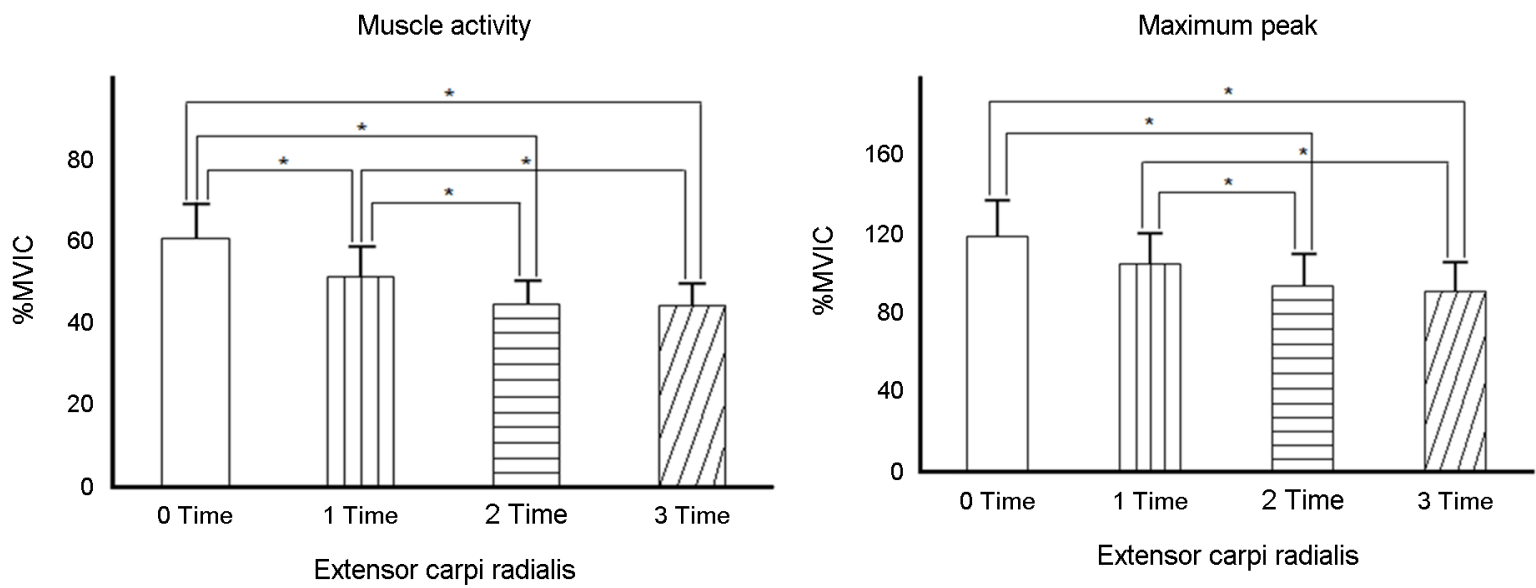

Figure 2. The comparison of muscle activity (left) and maximum peak (right) of ECR muscle during the 4 taping conditions

\section{Discussion}

This study was designed to identify changes in the muscle activities and maximum peak of the ECR muscle after repetitive taping applications, and to compare the effects of these 4 methods. Our findings suggest that ET may be used favorably to improve muscle activity and maximum peak; however, we did not find significant differences between applying taping twice and thrice. For the functional impairments in rehabilitation, which involves improving the performance of UE and thereby enhancing the activity of daily living, appropriate UE movement patterns are required. ET has been widely adopted to the optimization of UE function in various therapeutic methods by many rehabilitation clinicians. Although studies to support the therapeutic benefits of ET in the management of symptoms of a variety of neuromuscular disorders have been conducted previously (Mostafavifar et al., 2012), differences in muscle activity with a different number of taping application have not yet been sufficiently investigated.

In this study, EMG measurements were performed with the UE in a static position during an isometric contraction while holding a specific weight. This method has been acceptable for most clinicians, as performing measurements in a dynamic state, which may be considered more functional, requires special devices to control the dynamic movement. Our findings showed that the muscle activities of the ECR muscle improved by up to 27\%MVIC (from $60.70 \pm 7.89$ to $44.27 \pm 6.22$ ) between no taping and applying taping thrice. Similarly, the maximum peak of the ECR muscle improved by up to $24 \% \mathrm{MVIC}$ (from $118.7 \pm 13.62$ to $90.6 \pm 12.33$ ) between the no taping and applying taping thrice. However, the muscle activities and maximum peak of the ECR

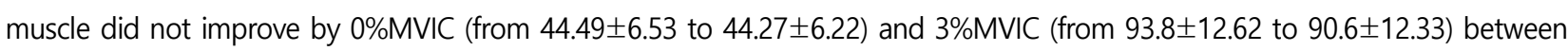
applying taping twice and thrice, respectively. This study was conducted to investigate the effects of repeated taping of the ECR muscle, and whether the effects of taping on muscle activity and maximum peak may be related to neuromuscular control and proprioceptive feedback factors.

The neurophysiological mechanisms underlying the action of the ET are not clear yet. However, it may exert an effect in three 
ways as follows. First, the cutaneous fusimotor reflex activates gamma motor neurons, which induces a contraction of the muscular system (Gómez-Soriano et al., 2014). Although the reflex is not strong, it is sufficient to continuously contract the muscles. Second, the appropriated stimulus of the taping on the skin may occur in a vasomotor nerve reflex, thereby increasing acetylcholine and histamine secretion levels, improving the blood and lymphatic circulation. Third, gate control theory asserts that activation of nerves does not transmit pain signals, called non-nociceptive fibers, and can interfere with signals from pain fibers, thereby inhibiting pain (Alexander et al., 2008).

Many studies have provided evidence for the favorable effects of taping. The ET has been reported to result in flexibility and muscle strengthening (Vicenzino et al., 2003), as well as an elasticity of $130-140 \%$ of the length of the ET in the case of dynamic movement without discomfort. The contractile force of the ET has been reported to increase the space made between the skin and muscle layer, improving blood circulation, and lymph and tissue fluid flow; increasing fascia formation; and removing waste products, which in turn reduces the pressure within the dermis (Morris et al., 2013). Furthermore, it activates proprioception by providing a strong stimulation to the skin and promoting muscle spindle sensitivity. In this way, the ET increases the contractile response of the muscle (Refshauge et al., 2000).

In clinical application, the elasticity of the tape can be adjusted and fixed in accordance with the muscle status. The effect was observed in patients with different neuromuscular disorders. Taping has recently become a common treatment of muscular dysfunction and joint pain (Kaya et al., 2011; Williams et al., 2012). Instead of repeated taping, the color of the ET is matched with that of common skin types after three applications. The blue tape is used for acute diseases such as sprains and strain, and the red tape is used for chronic disease. The color of the ET is simply used to distinguish an acute condition from a chronic condition. The commonly used ET diameter is $7.5,5,3.75$, or $2.5 \mathrm{~cm}$. The ET can be $1-$, Y-, or standard-shaped.

However, as found in this study, the changes observed did not significantly differ between applying taping twice and thrice in the ECR muscle. This indicates that the efficacy of applying taping thrice in the ECR muscle is not clear. However, it is hard to adapt the method in each individual muscles and the forearm. In addition, during EMG measurement, the crosstalk caused by adjacent muscles may disturb the EMG signal obtained from the ECR muscle, which makes EMG data less accurate. Taping is probably related to shortening of muscle length, placing it in a more mechanically advantageous length or alignment, thereby leading to mechanical improvement of sensory-motor function and proprioception (Van Elk et al., 2004). Unlike our study, one previous study reported a positive effect of three applications of ET on gripping force and muscle strength in 15 adult women with tennis elbow (Jaraczewska and Long, 2006; Shamsoddini and Hollisaz, 2013). However, the findings after two and three applications were not compared. Our study findings showed a tendency for improvement in the muscle activity and maximum peak of ECR muscle, from no taping to two tapings. Therefore, the results of this study should prompt further investigation of the effects of the number of taping of the different muscles on muscle activity and maximum peak.

We acknowledge that this study has several limitations. First, the small number of subjects largely limited the generalizability of our results. Second, our study focused only on the initial effect after the taping intervention and not on the maintenance effect. The long-term effect of the intervention needs to be studied in a future research. Lastly, this study included EMG data obtained only from the ECR muscle of the UE. Thus, further studies are needed with a larger sample size, longer duration, and other involved muscles of the body, which can be evaluated to describe the possible mechanisms of neuromuscular control.

\section{Conclusion}

The ET has generally been used to improve the neuromuscular and musculoskeletal systems in clinical settings by affecting muscle activity. It may be an advantageous option for the encouragement of therapeutic strategies for functional improvement. This study aimed to evaluate the differences in muscle activity and maximum peak value between no taping, and applying taping once, twice, 
and thrice, and to compare the effects of these ET methods. Our findings indicated that repeated taping application up to two times may be useful in improving the efficacy of the activity and maximum peak value of the ECR muscle. This study provides fundamental information to future researchers regarding the effect of repeated ET application on muscle activity of the human body.

\section{Acknowledgements}

This work (Grants No. C0146633) was supported by Business for Cooperative R\&D between Industry, Academy, and Research Institute funded Korea Small and Medium Business Administration in 2013.

\section{References}

Alexander, C.M., McMullan, M. and Harrison, P.J., What is the effect of taping along or across muscle on motor neuron excitability? A study using triceps surae, Manual therapy, 13(1), 57-62, 2008.

Bartolo, M., De Nunzio, A.M., Sebastiano, F. et al., Arm weight support training improves functional motor outcome and movement smoothness after stroke, Functional neurology, 29(1), 15-21, 2014.

Bell, A. and Muller, M., Effects of kinesio tape to reduce hand edema in acute stroke, Topics in stroke rehabilitation, 20(3), 283288, 2013.

Buckle, P.W. and Devereux, J.J., The nature of work-related neck and upper limb musculoskeletal disorders, Applied ergonomics, 33(3), 207-217, 2002.

Chang, H.Y., Wang, C.H., Chou, K.Y. et al., Could forearm Kinesio Taping improve strength, force sense, and pain in baseball pitchers with medial epicondylitis?, Clinical journal of sport medicine, 22(4), 327-333, 2012.

Cram, J.R., Kasman, G.S. and Holtz, J.H., Introduction to surface Electromyography, Gaithersburg, MD: Aspen Publishers Inc., 304320, 1998.

Da Costa, C.S., Rodrigues, F.S., Leal, F.M. et al., Investigating the effects of Kinesio Taping ${ }^{\circledR}$ on functional activities in children with cerebral palsy: Pilot study, Developmental neurorehabilitation, 16(2), 121-128, 2013.

Firth, B.L., Dingley, P., Davies, E.R. et al., The effect of kinesiotape on function, pain, and motoneuronal excitability in healthy people and people with Achilles tendinopathy, Clinical journal of sport medicine, 20(6), 416-421, 2010.

Haudum, A., Birklbauer, J. and Müller, E., The influence of external perturbations on running kinematics and muscle activity before and after accommodation, Journal of sports science \& medicine, 11(4), 582-591, 2012.

Gómez-Soriano, J., Abián-Vicén, J., Aparicio-García, C. et al., The effects of Kinesio taping on muscle tone in healthy subjects: a double-blind, placebo-controlled crossover trial, Manual therapy, 19(2), 131-136, 2014.

Jaraczewska, E. and Long, C., Kinesio Taping in Stroke: Improving functional use of the upper extremity in hemiplegia, Topics in stroke rehabilitation, 13(3), 31-42, 2006. 
Karadag-Saygi, E., Cubukcu-Aydoseli, K., Kablan, N. et al., The role of kinesiotaping combined with botulinum toxin to reduce plantar flexors spasticity after stroke, Topics in stroke rehabilitation, 17(4), 318-322, 2010.

Kaya, E., Zinnuroglu, M. and Tugcu, I., Kinesio taping compared to physical therapy modalities for the treatment of shoulder impingement syndrome, Clinical rheumatology, 30(2), 201-207, 2011.

Kendall, F.P., McCreary, E.K., Provance, P.G. et al., Muscle: Testing and function, with posture and pain. 5th ed. Baltimore, MD, Lippincott Williams and Wilkins, 17-330, 2005.

Montalvo, A.M., Cara, E.L. and Myer, G.D., Effect of kinesiology taping on pain in individuals with musculoskeletal injuries: Systematic review and meta-analysis, The Physician and sports medicine, 42(2), 48-57, 2014.

Morris, D., Jones, D., Ryan, H. et al., The clinical effects of Kinesio ${ }^{\circledR}$ Tex taping: A systematic review, Physiotherapy theory and practice, 29(4), 259-270, 2013.

Mostafavifar, M., Wertz, J. and Borchers, J., A systematic review of the effectiveness of kinesio taping for musculoskeletal injury, The Physician and sports medicine, 40(4), 33-40, 2012.

Pandian, J.D., Kaur, P., Arora, R. et al., Shoulder taping reduces injury and pain in stroke patients: randomized controlled trial, Neurology, 80(6), 528-532, 2013.

Parreira Pdo, C., Costa Lda, C., Hespanhol Junior, L.C. et al., Current evidence does not support the use of Kinesio Taping in clinical practice: a systematic review, Journal of physiotherapy, 60(1), 31-39, 2014.

Refshauge, K.M., Kilbreath, S.L. and Raymond, J., The effect of recurrent ankle inversion sprain and taping on proprioception at the ankle, Medicine and science in sports and exercise, 32(1), 10-15, 2000.

Shamsoddini, A. and Hollisaz, M.T., Effects of taping on pain, grip strength and wrist extension force in patients with tennis elbow, Trauma monthly, 18(2), 71-74, 2013.

Smith, M., Sparkes, V., Busse, M. et al., Upper and lower trapezius muscle activity in subjects with subacromial impingement symptoms: is there imbalance and can taping change it? Physical therapy in sport, 10(2), 45-50, 2009.

Van Elk, N., Faes, M., Degens, H. et al., The application of an external wrist extension force reduces electromyographic activity of wrist extensor muscles during gripping, The Journal of orthopaedic and sports physical therapy, 34(5), 228-234, 2004.

Vicenzino, B., Brooksbank, J., Minto, J. et al., Initial effects of elbow taping on pain free grip strength and pressure pain threshold, The Journal of orthopaedic and sports physical therapy, 33(7), 400-407, 2003.

Williams, S., Whatman, C., Hume, P.A. et al., Kinesio taping in treatment and prevention of sports injuries: a meta-analysis of the evidence for its effectiveness, Sports medicine, 42(2), 153-164, 2012. 


\section{Author listings}

Ju Chul Cho: bbangil11@kyuh.ac.kr

Highest degree: MSc, Department of Physical Therapy, Konyang University

Position title: Supervisors, Rehabilitation Team, Konyang University Hospital

Areas of interest: Human Movement Analysis, Balance Disorders

Byoung Kwon Lee: Ibk6326@konyang.ac.kr

Highest degree: PhD, Department of Sport Rehabilitation, Koln University, Germany

Position title: Assistant Professor, Department of Physical Therapy, Konyang University

Areas of interest: Sport Physical Therapy, Musculoskeletal Exercise

Seung Chul Chon: biomechanic1@hotmail.com, keyjune@konyang.ac.kr

Highest degree: PhD, Department of Physical Therapy, Yonsei University

Position title: Assistant Professor, Department of Physical Therapy, Konyang University

Areas of interest: Physical Examination, Neuromusculoskeletal Disorders 